\title{
Tendencias en el uso de la tierra y diversidad productiva en establecimientos agropecuarios del centro-sur de la provincia de Buenos Aires (Argentina)
}

Requesens, E. y L. Silva

\begin{abstract}
RESUMEN
El partido de Benito Juárez, ubicado en el centro-sur de la provincia de Buenos Aires (Argentina), es ambientalmente heterogéneo y productivamente mixto, con tradición predominantemente ganadera. A fin de evaluar las tendencias en el uso de la tierra, se describió la evolución de los diferentes rubros productivos y su diversidad durante el periodo 1998-2007, sobre la base de una selección de 30 establecimientos. El pastizal natural ocupó la mayor proporción de superficie promedio en el período considerado, pero reflejó una pérdida de 23 puntos porcentuales compensada sólo parcialmente por un aumento de pasturas perennes. La suma de ambos recursos forrajeros evidenció una caída en la superficie destinada a ganadería y, al final del periodo, fue ligeramente superada por la superficie destinada a cultivos anuales. Entre éstos, la soja fue el cultivo con mayor expansión pero su participación relativa alcanzó sólo el $11,02 \%$. Paralelamente, la diversidad productiva, estimada mediante una adaptación del índice de diversidad de Shannon, aumentó 30,84\%. Aunque es evidente el avance de cultivos anuales en desmedro de pastizales naturales, el reemplazo parcial de estos últimos por pasturas perennes y el aumento en la diversidad productiva limitan los riesgos ambientales potenciales, puestos de manifiesto en otras regiones del país.
\end{abstract}

Palabras clave: Buenos Aires, expansión agrícola, sojización, riesgos ambientales.

Requesens, E. and L. Silva, 2011. Tendencies in land use and productive diversity in central-south farms of the Province of Buenos Aires (Argentina). Agriscientia XXVIII: 75-83

\section{SUMMARY}

Benito Juarez county, in the central-south Buenos Aires province (Argentina), is environmentally and productively heterogeneous, with predominance of cattle 
farming. In order to evaluate the tendencies in land use, the evolution of different productive items and their diversity throughout the period 19982007 were described on the basis of 30 farms selected. The grasslands occupied the greater proportion of the area throughout the period studied, but they reflected a loss of 23 percentage points which was only partially compensated by an increase of perennial pastures. This fact caused a decrease in the area destined to cattle raising which at the end of the period was slightly surpassed by the area destined to annual crops. Among the latter, soybean was the crop with greater expansion but its relative participation reached only $11.02 \%$. Simultaneously, productive diversity, estimated by an adaptation of Shannon's diversity index, increased 30.84\%. Although the advance of annual crops to the detriment of grasslands is evident, the partial replacement of the latter for perennial pastures and the increase in productive diversity limit the potential environmental risks revealed in other regions of the country.

Key words: Buenos Aires, agricultural expansion, soybean expansion, environmental risks.

E. Requesens. Facultad de Agronomía (UNICEN), República de Italia 780, Azul, Argentina. L. Silva. Licenciatura en Diagnóstico y Gestión Ambiental, Facultad de Ciencias Humanas (UNICEN), Campus Universitario Tandil. Correspondencia a E. Requesens: erequese@faa.unicen.edu.ar

\section{INTRODUCCIÓN}

El proceso de "agriculturización" ha sido definido como el uso creciente y continuo de las tierras para cultivos agrícolas en reemplazo de usos ganaderos o mixtos. Desde la perspectiva de la región pampeana, se lo vincula a cambios tecnológicos, intensificación ganadera, expansión de la frontera agropecuaria hacia regiones extrapampeanas y tendencia al monocultivo de soja o la combinación trigo-soja (Manuel-Navarrete et al., 2005). Como resultado de este proceso, entre 1990 y 2006 la superficie de nuestro país destinada a agricultura experimentó un incremento de $45 \%$ y la diversidad de cultivos sufrió una caída superior a 20\%. Al final de dicho periodo, la soja llegó a ocupar la mitad de la superficie cultivada en una clara tendencia a la homogeneización del paisaje agrícola (Aizen et al., 2009).

Con respecto a la región pampeana en particular, Ramírez y Porstmann (2008) analizaron la dinámica del área sembrada con los cuatro cultivos principales: soja, girasol, maíz y trigo, durante el ciclo comprendido entre las campañas 1980/1981 y 2006/2007. En conjunto, la superficie sembrada creció de 13,5 millones de hectáreas al comienzo del ciclo a 27,8 millones en la última campaña. La tasa promedio de incremento en la superficie total fue de $3,90 \%$ anual y la de producción de 8,88\%. Esto último evidencia que, además de una expansión en superficie, tuvo lugar un aumento en la productividad.

Paralelamente, la extrapolación del modelo productivo pampeano a regiones extrapampeanas ha profundizado la deforestación en estas últimas con síntomas evidentes de insustentabilidad ambiental, socio-cultural y productiva (Manuel-Navarrete et al., 2005). El total deforestado entre 1998 y 2006 asciende a 2.295.567 ha (Secretaría de Ambiente y Desarrollo Sustentable de la Nación, 2008). La región del Chaco argentino es la más afectada. En la provincia de Santiago del Estero, la superficie agrícola se incrementó siete veces entre 1988 y 2003 (Pérez Carrera et al., 2008). Si se considera el área correspondiente al Chaco interior en su conjunto, la superficie agrícola se expandió $78,74 \%$ entre 1992 y 2002, con máximos de 85,17\% en el Chaco subhúmedo central (Torrella et al., 2005).

Los costos ambientales de la expansión agrícola se manifiestan en diversos aspectos vinculados a la degradación del suelo, contaminación del agua y del aire, pérdida de diversidad biológica y deterioro de servicios ecológicos esenciales (FAO, 2007; Viglizzo, 2008). A ellos, deben sumarse los costos económicos asociados a un aumento en la 
vulnerabilidad de la empresa agropecuaria frente a la inestabilidad del clima y del mercado (Viglizzo, 1994), además de los costos sociales derivados de los cambios en la estructura productiva y régimen de tenencia de la tierra que operaron, con características particulares, tanto en la región pampeana como en regiones extrapampeanas (González y Román, 2009).

En el centro-sur de la provincia de Buenos Aires, Argentina, se ubica el partido de Benito Juárez, que se expande entre $59^{\circ}$ y $60^{\circ} \mathrm{O}$ y entre $37^{\circ} 10^{\prime}$ y 37058' S con una altitud promedio de $199 \mathrm{msnm}$. Posee una superficie de 530.000 ha y representa el $1,72 \%$ del territorio bonaerense. Su elevada heterogeneidad ambiental y una tradición fuertemente ganadera, lo convierten en un área de estudio interesante para evaluar las tendencias en el uso de la tierra durante los últimos años, a la luz del proceso de agriculturización comentado previamente.

En el contexto del presente trabajo, la expresión "uso de la tierra" se refiere a la utilización del suelo para diversos rubros productivos tanto en la dimensión espacial como en la temporal (Altieri, 1999). Por su parte, la variedad de rubros productivos y su participación relativa en la ocupación de la superficie, son utilizados como una expresión de la diversidad productiva y constituyen una herramienta sumamente valiosa para interpretar la estructura de los sistemas de producción y su relación con diversos mecanismos homeostáticos que regulan su funcionamiento. Entre los beneficios resultantes de la diversificación se pueden mencionar el aumento de la productividad, estabilidad y sustentabilidad de los sistemas productivos, la conservación de los recursos naturales, particularmente el suelo, y la reducción de los riesgos económicos.

Pese a estos beneficios, y de acuerdo a lo sucedido en la región pampeana en su conjunto y en regions extrapampeanas, se espera que los establecimientos agropecuarios del partido de Benito Juárez registren un proceso de expansión agrícola con pérdida de diversidad productiva como consecuencia de la concentración en el cultivo de soja, en detrimento de cultivos tradicionales y de pasturas implantadas y pastizales naturales destinados a las actividades ganaderas.

En el contexto planteado, los objetivos de este trabajo fueron: a) describir la evolución temporal de los diferentes rubros productivos a escala de establecimientos agropecuarios en el partido de Benito Juárez, b) calcular y describir la evolución temporal de la diversidad productiva, y c) discutir los probables riesgos ambientales de las tendencias observadas.

\section{MATERIALES Y METODOS}

\section{Características del área de estudio}

El clima regional es del tipo templado pampeano con una temperatura media anual de $14-15{ }^{\circ} \mathrm{C}$ y un promedio de precipitaciones anuales de 1030 $\mathrm{mm}$, mayormente concentradas entre octubre y marzo.

El territorio del partido de Benito Juárez es ambientalmente heterogéneo con sectores representativos de tres subregiones de la Pampa Húmeda: el sistema orográfico de Tandilia hacia el norte, la Pampa Interserrana hacia el sudeste y la Pampa Deprimida hacia el oeste del partido. En el contexto de esta diversidad ambiental, han sido diferenciados 14 sectores en base a la capacidad de uso de las tierras (Sociedad Rural de Juárez, 2007). De dicho trabajo se desprende que sólo un área reducida del territorio juarense $(19,15 \%)$ posee aptitud agrícola o agrícola-ganadera, mientras que el resto posee tierras con capacidades de uso ganadera $(44,76 \%)$ o ganadera-agrícola (36,08\%).

Las características descritas hacen del partido de Benito Juárez un territorio de explotación mixta, con predominio de la actividad ganadera. En 2006, el stock bovino era de 405.046 cabezas. En agricultura, sobresalen los cultivos de soja con 47.500 ha sembradas y 102.565 tn de producción y el cultivo de trigo con 45.000 ha sembradas y 167.960 tn de producción en la campaña 2006/2007. Le siguen el girasol con una superficie de 15.500 ha y una producción de 23.840 tn y el maíz con una superficie de 7.000 ha y una producción de 15.600 tn en la misma campaña (CITAB, 2009).

En cuanto a la estructura agraria, el partido posee un total de 513 establecimientos, de los cuales, 33\% posee una superficie inferior a las 200 ha, $23 \%$ posee una superficie entre 200 y 500 ha, $21,5 \%$ entre 500 y 1000 ha, y el 22,5\% posee una superficie mayor a 1000 ha (CNA 2002).

\section{Obtención y análisis de los datos}

El universo de estudio abarcó a los establecimientos agropecuarios del partido de Benito Juárez, excepto aquellos ubicados en sectores con capacidad de uso limitada a la ganadería. Sobre este universo, se procedió a seleccionar una muestra de un número determinado de establecimientos, cada uno de los cuales se constituyó en una fuente de información. La elección de los establecimientos como unidad de análisis permite obtener datos más confiables que los censos o estimaciones a nivel de partido y facilita la tipificación de los sistemas productivos. El periodo de estudio abarcó 10 
años entre 1998 y 2007 inclusive.

Para la obtención de la muestra se recurrió a consultas y entrevistas con diferentes actores vinculados al medio rural: asesores técnicos, agencia de extensión del INTA, asociaciones de productores, etc. Finalmente, fueron seleccionados 30 establecimientos representativos que contaban con información confiable, accesible y adecuada a los objetivos del trabajo. A fin de recabar tal información, se confeccionó una planilla de doble entrada (lote $x$ año) para cada establecimiento, las cuales fueron llenadas por sus referentes o responsables (propietarios, administradores, encargados o asesores). La superficie de cada lote fue explicitada junto a la identificación de estos últimos y los rubros productivos correspondientes fueron incorporados en las casillas del interior de la planilla.

El conjunto de los establecimientos seleccionados abarcó una amplia gama de tamaños entre 24 y 2371 ha. El $20 \%$ posee una superficie inferior a 200 ha, $27 \%$ entre 200 y 500 ha, $40 \%$ entre 500 y 1000 ha y $13 \%$ más de 1000 ha. Al no considerarse los establecimientos de sectores restringidos a la ganadería, la proporcionalidad de diferentes rangos de tamaño no se ajustó estrictamente a la mencionada para el partido. Al comienzo del periodo de estudio, 16 establecimientos realizaban diferentes combinaciones de actividades agrícolas y ganaderas mientras que, entre los restantes, 12 realizaban ganadería pura y 2 agricultura pura.

Las principales variables de análisis fueron: 1) la superficie anual destinada a los diferentes rubros productivos y 2) la diversidad productiva asociada al número y superficie relativa de estos últimos. Los rubros productivos fueron diferenciados en primera instancia en los que presentan ciclo anual y aquellos basados en vegetación perenne. Los primeros incluyeron a todos los cultivos anuales, tanto los destinados exclusivamente a cosecha de granos (trigo, soja, girasol) como aquellos que suelen destinarse a doble propósito, pastoreo y/o cosecha para grano o silo, como maíz, sorgo y avena. Los segundos incluyeron a pastizales naturales y pasturas perennes implantadas que, en conjunto, constituyen la base forrajera de los sistemas ganaderos. Para cada año se calcularon los valores promedio de cada rubro y su proyección a lo largo del periodo considerado permitió analizar las tendencias en el uso de la tierra.

Sobre la base del porcentaje de la superficie ocupada por cultivos anuales, los establecimientos fueron clasificados de acuerdo a los tipos de sistemas productivos propuestos por Alesandria et al. (2001) y cuyo detalle se presenta en la Tabla 1. Cabe aclarar que, en el caso del presente trabajo, y ante la imposibilidad de cuantificar correctamente los diferentes usos en cultivos doble propósito, toda la superficie ocupada por cultivos anuales, independientemente de su destino final, fue considerada superficie agrícola.

Tabla 1: Clasificación de sistemas productivos de acuerdo a Alesandria et al .(2001).

\begin{tabular}{|c|c|}
\hline Tipo de sistema productivo & $\begin{array}{l}\text { Porcentaje de superficie } \\
\text { con cultivos anuales }\end{array}$ \\
\hline Agrícolas puros & Mayor a $99 \%$ hasta $100 \%$ \\
\hline Agrícolas complementados & Mayor a $75 \%$ hasta $99 \%$ \\
\hline $\begin{array}{l}\text { Mixtos predominantemente } \\
\text { agrícolas }\end{array}$ & Mayor a $60 \%$ hasta $75 \%$ \\
\hline Mixtos equilibrados & Mayor a $40 \%$ hasta $60 \%$ \\
\hline $\begin{array}{l}\text { Mixtos predominantemente } \\
\text { ganaderos }\end{array}$ & Mayor a $25 \%$ hasta $40 \%$ \\
\hline Ganaderos complementados & Mayor a $1 \%$ hasta $25 \%$ \\
\hline Ganaderos puros & Mayor a $0 \%$ hasta $1 \%$ \\
\hline
\end{tabular}

Para estimar el nivel de diversidad productiva se apeló a la utilización del índice de diversidad de Shannon. En su aplicación a comunidades vegetales, este índice considera la contribución de cada especie presente en la comunidad ponderada por la abundancia relativa. La ecuación correspondiente se expresa como

$H^{\prime}=-\Sigma_{\mathrm{i}=1}^{\mathrm{s}}\left(\mathrm{p}_{\mathrm{i}}\right)\left(\log \mathrm{p}_{\mathrm{i}}\right)$

donde $H$ 'es el valor de diversidad, s es el número de especies y $p_{i}$ es la proporción o abundancia relativa de la especie $i$ (Begon et al., 1995). En su utilización como estimador de diversidad productiva, los rubros productivos reemplazaron a las especies y su abundancia relativa fue ponderada por la superficie ocupada por cada uno de ellos. A partir de los valores de $\mathrm{H}$ obtenidos para cada establecimiento, se calculó la diversidad promedio para cada año y su proyección gráfica permitió analizar su tendencia a lo largo del periodo estudiado.

\section{RESULTADOS}

En la Tabla 2 se presenta, en valores absolutos promedio, la superficie asignada a los diferentes rubros productivos que se realizan en la muestra de establecimientos seleccionados, ordenados alfabéticamente, además de las sumas de rubros anuales y perennes y la superficie total por establecimiento. Esta última experimentó un crecimiento promedio de entre 20 y 27 ha en la segunda mitad del periodo estudiado, producto de la ampliación territorial de algunos establecimientos. Entre los rubros productivos es posible reconocer dos grandes grupos. Por un lado, los rubros considerados 
principales por haber sido registrados todos los años y conformar la base de la estructura productiva de los establecimientos estudiados. Este grupo incluye al pastizal natural, que ocupa una fracción importante de la superficie y constituye el principal sustento de la ganadería de cría vacuna, las pasturas implantadas perennes y varios cultivos anuales como avena, girasol, maíz, soja y trigo. Por otro lado, están los rubros secundarios como alpiste, cebada, soja de segunda y sorgo, los cuales no han sido registrados todos los años y ocupan superficies de escasa relevancia en comparación a los anteriores.

Considerando el grupo de rubros principales, la proyección gráfica de los datos de la Tabla 2, expresados ahora en términos relativos, permite

Tabla 2: Evolución de la superficie promedio de los diferentes rubros productivos en el partido de Benito Juárez. Valores expresados en hectáreas (ha). Pastura perenne se refiere a la siembra de especies forrajeras con ciclo de crecimiento superior a dos años, solas o en mezclas. Soja de segunda se refiere a la soja sembrada inmediatamente sobre rastrojos de cultivos antecesores de ciclo invernal.

\begin{tabular}{lrrrrrrrrrr}
\hline RUBRO PRODUCTIVO & 1998 & 1999 & 2000 & 2001 & 2002 & 2003 & 2004 & 2005 & 2006 & 2007 \\
\hline Alpiste & & 1,67 & 2,83 & & 2,33 & & & & & \\
Avena & 25,17 & 19,97 & 26,00 & 31,33 & 23,13 & 19,00 & 33,03 & 5,63 & 20,77 & 19,13 \\
Cebada & 3,50 & & & & & 1,67 & 2,87 & 3,13 & 4,93 & 25,60 \\
Girasol & 32,6 & 46,03 & 36,90 & 38,17 & 38,80 & 26,00 & 19,60 & 22,13 & 19,30 & 60,23 \\
Maíz & 28,84 & 20,83 & 24,30 & 24,93 & 26,73 & 20,77 & 19,47 & 33,00 & 50,27 & 37,17 \\
Pastizal natural & 334,30 & 322,07 & 309,37 & 291,93 & 279,60 & 270,10 & 261,57 & 239,30 & 229,33 & 219,46 \\
Pastura perenne & 33,80 & 39,90 & 47,33 & 54,80 & 67,63 & 71,20 & 73,13 & 79,97 & 85,77 & 84,93 \\
Soja & 20,67 & 17,17 & 24,57 & 33,83 & 36,20 & 78,30 & 53,47 & 81,13 & 64,37 & 63,13 \\
Soja de segunda & & & & & 1,33 & & & 0,80 & & 3,43 \\
Sorgo & & & 0,93 & 0,93 & 2,33 & 4,13 & 0,83 & 4,37 & 4,87 & 4,60 \\
Trigo & 67,10 & 78,33 & 73,40 & 70,03 & 80,03 & 74,53 & 106,60 & 94,13 & 91,40 & 55,30 \\
Suma de cultivos anuales & 177,87 & 184,00 & 189,27 & 199,23 & 217,07 & 224,40 & 231,00 & 246,43 & 257,90 & 268,60 \\
Pastizal natural + pastura & 368,10 & 361,97 & 356,70 & 346,73 & 341,07 & 341,30 & 334,70 & 319,27 & 315,10 & 304,40 \\
perenne & & & & & & & & & & \\
Total establecimiento & 545,97 & 545,97 & 545,97 & 545,97 & 558,13 & 565,70 & 565,70 & 565,70 & 573,00 & 573,00 \\
\hline
\end{tabular}

visualizar mejor las tendencias a lo largo del periodo analizado (Figura 1). Resulta evidente que, a lo largo de todo el período, el pastizal natural ocupó la mayor proporción de superficie pero con una tendencia permanentemente declinante. Paralelamente, otros rubros incrementaron su participación, entre los cuales se destacan las pasturas implantadas y la soja. El resto de los cultivos presentaron fluctuaciones anuales alrededor de valores medios. Entre estos últimos, el trigo mantuvo su predominio pero, hacia el final del ciclo, mostró una tendencia declinante dando lugar a una equiparación con la soja y el girasol.

La caída en la superficie de pastizales naturales fue en parte compensada por un aumento en la implantación de pasturas perennes. Sin embargo, ello no resultó suficiente para evitar la reducción de la superficie destinada a conformar la base de sustentación de la ganadería. La suma de ambos recursos forrajeros evidenció una caída a lo largo del periodo de estudio que fue compensada por

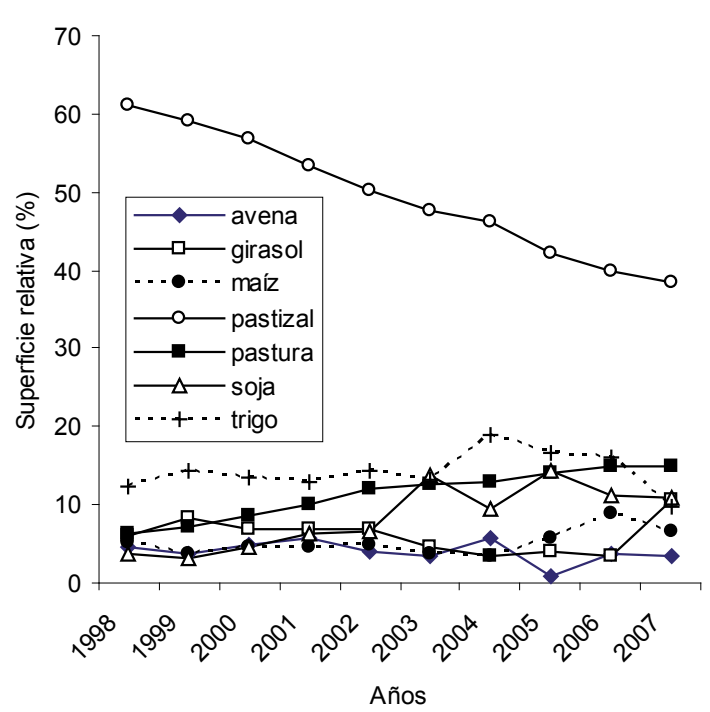

Figura $\mathbf{N}^{\circ}$ 1: Tendencia de la superficie relativa (porcentaje de la superficie total) de los rubros productivos principales en el partido de Benito Juárez. 
el aumento progresivo de la superficie destinada a cultivos anuales (Figura 2). Al final del periodo estudiado, esta última sobrepasó el $50 \%$ y superó levemente a los recursos forrajeros perennes.

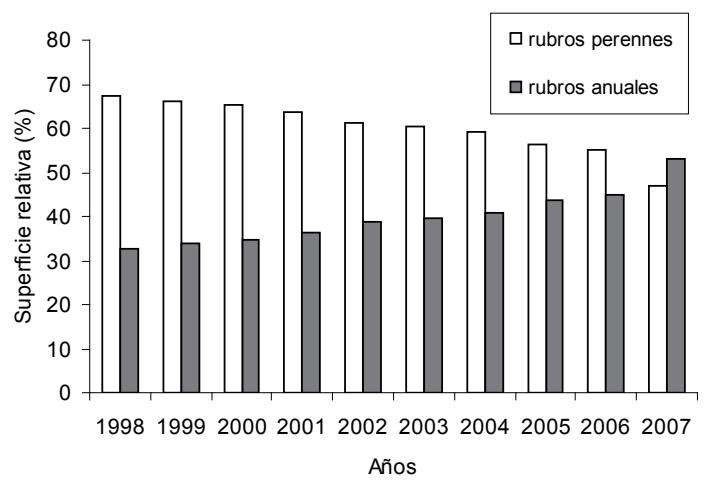

Figura 2: Tendencias de las superficies relativas (porcentaje de la superficie total) de rubros anuales (cultivos para granos + cultivos doble propósito) y perennes (pastizal natural + pastura perenne) a lo largo del periodo de estudio en el partido de Benito Juárez.

Las tendencias observadas en el uso de la tierra tuvieron como resultado cambios en la importancia relativa de diferentes tipos de sistemas productivos. Al comienzo del periodo de estudio se registró un claro predominio de sistemas ganaderos puros $(36,67 \%)$ los que, junto a los ganaderos complementados y mixtos predominantemente ganaderos, representaban el 56,67\% (Figura 3). Al final del periodo, la representación de sistemas ganaderos puros descendió al 20\% y el conjunto de tipologías con predominio ganadero representaba el $40 \%$;

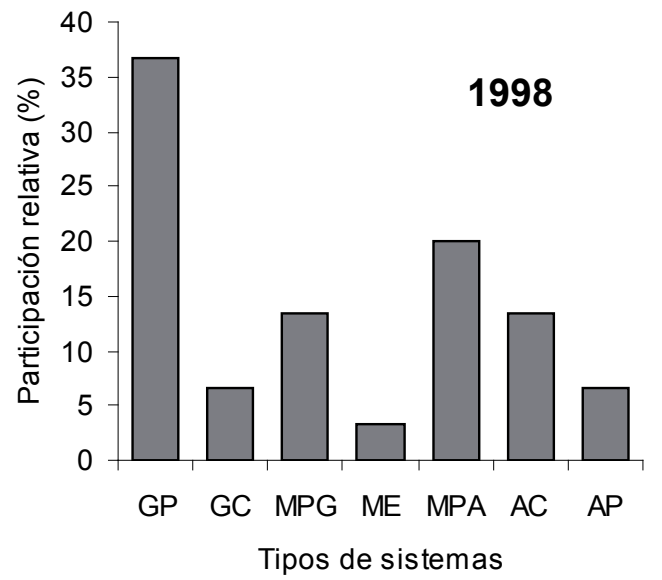

Figura 3: Participación relativa (porcentaje del número total de establecimientos) de los distintos tipos de sistemas productivos en el partido de Benito Juárez, en 1998. GP: ganadero puro GC: ganadero complementado - MPG: mixto predominantemente ganadero-ME: mixto equilibrado-MPA: mixto predominantemente agrícola - AC: Agrícola complementado - AP: agrícola puro. mientras que, al mismo tiempo, creció la representación de establecimientos con sistemas mixtos equilibrados y sistemas agrícolas puros, aunque éstos no superaron el 20\% (Figura 4).

Los resultados de la aplicación del índice de diversidad de Shannon como estimador de diversidad productiva a nivel de establecimiento, mostraron un rango de $\mathrm{H}^{\prime}$ entre 0 y 2,04. El ajuste por regresión a los valores promedio evidenció un incremento lineal cuya pendiente eleva la diversidad inicial de $H^{\prime}=0,6234$ (ordenada al origen) a $H^{\prime}=0,9014$ al final del periodo considerado, lo que representa un aumento de 30,84\% (Figura 5). Esta tendencia se explica fundamentalmente por la caída de los dos componentes más importantes al comienzo del período, pastizal natural y trigo, y el aumento de pasturas implantadas y soja (Tabla 2). Cabe destacar que la aplicación del coeficiente de correlación entre diversidad productiva $\left(\mathrm{H}^{\prime}\right)$ y tamaño de establecimiento no evidenció covariación entre ambas variables a lo largo de todo el periodo estudiado.

Al comparar la distribución de la superficie en los dos años extremos del periodo considerado (Tabla 3), se observa que la participación del pastizal natural disminuye casi 23 puntos porcentuales cayendo de $61 \%$ a $38 \%$. En menor medida, también el trigo y la avena bajaron su participación relativa. Por su parte, las pasturas implantadas y la soja con incrementos cercanos a 9 y 7 puntos porcentuales respectivamente, fueron los principales reemplazantes junto con el girasol y cebada los cuales incrementaron alrededor de 4 puntos su participación.

\section{DISCUSION}

Los establecimientos agropecuarios del partido de Benito Juárez no han sido ajenos al proceso de

Tabla 3: Participación relativa de los diferentes rubros productivos al comienzo y al final del periodo de estudio en el partido de Benito Juárez. Los valores expresan porcentajes de la superficie total.

\begin{tabular}{lccc}
\hline ACTIVIDAD & 1998 & 2007 & $\begin{array}{c}\text { Diferencia } \\
(2007-1998)\end{array}$ \\
\hline Pastizal natural & 61,23 & 38,30 & $-22,92$ \\
Trigo & 12,29 & 9,65 & $-2,64$ \\
Pastura perenne & 6,19 & 14,82 & 8,63 \\
Girasol & 5,97 & 10,51 & 4,54 \\
Maíz & 5,28 & 6,49 & 1,21 \\
Avena & 4,61 & 3,34 & $-1,27$ \\
Soja & 3,79 & 11,02 & 7,23 \\
Cebada & 0,64 & 4,47 & 3,83 \\
Soja de segunda & 0,00 & 0,60 & 0,60 \\
Sorgo & 0,00 & 0,80 & 0,80 \\
\hline
\end{tabular}




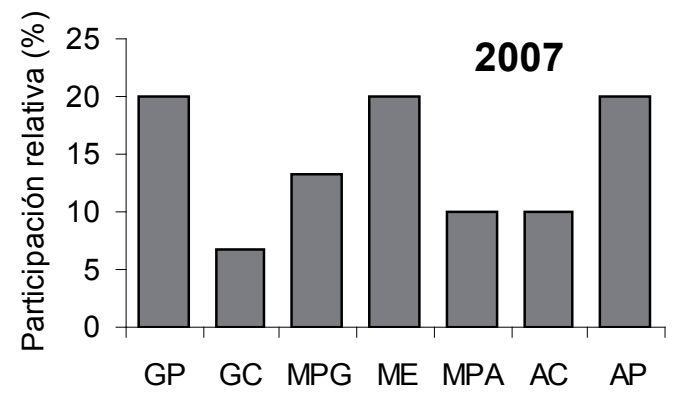

Tipos de sistemas

Figura 4: Participación relativa (porcentaje del número total de establecimientos) de los tipos de sistemas productivos en el partido de Benito Juárez, en 2007. GP: ganadero puro - GC: ganadero complementado - MPG: mixto predominantemente ganadero ME: mixto equilibrado - MPA: mixto predominantemente agrícola - AC: Agrícola complementado - AP: agrícola puro.

agriculturización ocurrido en otras áreas del país. Sin embargo, la expresión local de dicho proceso presenta particularidades que lo diferencian de lo ocurrido en otras áreas pampeanas y extrapampeanas. La información obtenida en el presente trabajo corrobora que, efectivamente, en la última década ha tenido lugar un proceso de expansión agrícola y "sojización" dentro del territorio juarense. No obstante, la intensidad de tal proceso resultó insuficiente para convertir un territorio inicialmente dominado por ganadería sobre pastizales naturales en otro totalmente transformado en agricultura. Los cambios en las tipologías resultaron en una distribución más uniforme de los diferentes sistemas productivos al final del periodo de estudio, a partir de una transformación de establecimientos ganaderos o con predominio ganadero en establecimentos mixtos equilibrados o con predominio agrícola. Las restricciones a la expansion agrícola pueden buscarse en la heterogeneidad ambiental del territorio juarense, dentro de la cual, la limitada disponibilidad de tierras con aptitud agrícola constituye una seria dificultad para la expansión de cultivos anuales, al menos con la tecnología disponible en la actualidad.

Utilizando índices comparables a los del presente trabajo, Pietrarelli (2009) analizó los cambios en el uso de la tierra en establecimientos de la región central de la provincia de Córdoba, entre las campañas 1997/1998 y 2003/2004. Esta autora registró un rango de $\mathrm{H}^{\prime}$ algo menor al registrado en el presente trabajo y una pérdida de diversidad productiva asociada a la expansión de la soja, cuya magnitud podría ser aún mucho mayor si se tomara como referencia a las etapas previas al lanzamiento masivo del paquete tecnológico asociado a la

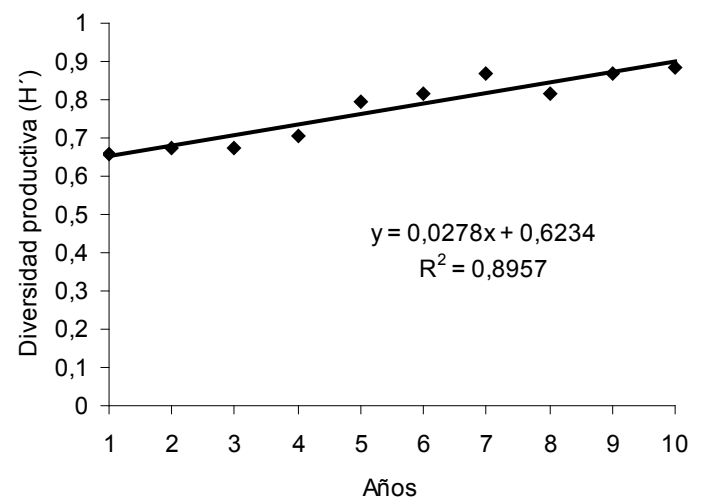

Figura $\mathbf{N}^{\circ}$ 5: Tendencia de la diversidad productiva, estimada a partir del índice de Shannon $\left(\mathrm{H}^{\prime}\right)$, a lo largo del periodo estudiado en el partido de Benito Juárez. En este caso, y a los fines del análisis de regresión, la denominación de los años sigue una secuencia de 1 a 10, donde el año 1 corresponde a 1998 y el año 10 corresponde a 2007

soja transgénica a mediados de los '90. Contrariamente, los cambios experimentados en el área de estudio del presente trabajo condujeron a un aumento en los niveles de diversidad productiva. Cabe destacar que el aumento registrado en esta última se debe tanto a un incremento en el número promedio de rubros productivos, que pasó de 2,57 en 1998 a 3,40 en 2007, como al aumento en la uniformidad en la distribución de superficie entre ellos. Esta última característica, estimada a partir del índice de equitatividad de Shannon (Begon et al., 1995), pasó de 0,54 en 1998 a 0,67 en 2007. Ello se explica, en parte, por el reemplazo parcial de pastizales naturales por pasturas implantadas y, en parte, porque la expansión de cultivos anuales no se concentró de manera notoria en la soja como ocurrió en general en la región pampeana. En ésta, la superficie de soja aumentó $738,5 \%$ entre las campañas 1980/1981 y 2006/2007, llegando a ocupar el $58,1 \%$ de la superficie cultivada al final del periodo (Ramírez y Porstmann, 2008).

Aunque es evidente el avance de cultivos anuales en desmedro de pastizales naturales, el reemplazo parcial de estos últimos por pasturas perennes y el aumento en la diversidad productiva permiten inferir que los riesgos ambientales potenciales, puestos de manifiesto en otras regiones del país, estarían limitados en el territorio de Benito Juárez. Sin embargo, no puede descartarse que en el futuro ocurran nuevos flujos de expansión agrícola sobre áreas de explotación mixta, y aun ganadera, motivados por precios relativos favorables, nuevos desarrollos tecnológicos y/o nuevos avances hacia formas contractuales "capital intensivo". En ese caso, podrían acelerarse algunos procesos que comprometen la sustentabilidad de los agroecosis- 
temas y que han sido ampliamente tratados en Viglizzo y Jobbágy (2010). En particular, se destacan la reducción de stock y desbalance de nutrientes esenciales como C, N y P (Frank y Viglizzo, 2010; Viglizzo, 2010), erosión del suelo y contaminación del ambiente (Viglizzo y Frank, 2010), pérdida de biodiversidad (Salvador, 2010) y deterioro de servicios ecológicos fundamentales como la regulación de agua, gases, nutrientes y clima, además de la provisión de hábitat y refugio para organismos benéficos (Carreño y Viglizzo, 2010). Todos estos procesos se verían potenciados, sobre todo, si el cultivo de soja siguiera aumentando su participación relativa dentro de los esquemas productivos (Pincén et al., 2010).

\section{BIBLIOGRAFÍA}

Aizen, M.A.; L.A. Garibaldi y M. Dondo, 2009. Expansión de la soja y diversidad de la agricultura argentina. Ecología Austral 19: 45-54.

Alessandria, E.; H. Leguía, L. Pietrarelli, J. Sánchez, S. Luque, M. Arborno, J. Zamar y D. Rubin, 2001. La agrodiversidad en sistemas extensivos. LEISA, Revista de Agroecología 16(4):10-11.

Altieri, M.A., 1999. Agroecología. Bases científicas para una agricultura sustentable. Nordan Comunidad. Montevideo. $338 \mathrm{pp}$.

Begon, M.; J.L. Harper y C.R. Townsend, 1995. Ecología. Individuos, poblaciones y comunidades. Ediciones Omega S.A., Barcelona. 886 pp.

Carreño L. y E. Viglizzo, 2010. Efecto de la agricultura sobre la provisión de servicios ecosistémicos. En: Viglizzo, E. y E. Jobbágy (Editores). Expansión de la frontera agropecuaria en Argentina y su impacto ecológicoambiental. Ediciones INTA, Buenos Aires. 102 pp.

CITAB (Centro de Investigaciones Territoriales y Ambientales Bonaerenses). Datos estadísticos e información general. www.bapro.com.ar/citab/estadisticas.htm. Consultado el 18/08/09.

CNA (Censo Nacional Agropecuario) 2002. www.indec. mecon.ar/agropecuario/cna. Consultado el 11/02/10.

FAO, 2007. El estado mundial de la agricultura y la alimentación. Parte 1: Pagos a los agricultores por servicios ambientales. Colección FAO: Agricultura $\mathrm{N}^{\circ}$ 38.

Frank F. y E Viglizzo, 2010. Balances de carbono, nitrógeno y fósforo. En: Viglizzo, E. y E. Jobbágy (Editores). Expansión de la frontera agropecuaria en Argentina y su impacto ecológico-ambiental. Ediciones INTA, Buenos Aires. 102 pp.

González, M. y M. Román, 2009. Expansión agrícola en áreas extrapampeanas de la Argentina. Una mirada desde los actores sociales. Cuadernos de Desarrollo Rural, Bogotá (Colombia), 6: 99-120.

Manuel-Navarrete, D.; G. Gallopin, M. Blanco, M. Díaz-
Zorita, D. Ferraro H. Herzer, P. Laterra, J. Morello, M.R. Murmis, W. Pengue, M. Piñeiro, G. Podestá, E.H. Satorre, M. Torrent, F. Torres, E. Viglizzo, M.G. Caputo, A. Celis, 2005. Análisis sistémico de la agriculturización en la pampa húmeda argentina y sus consecuencias en regiones extra-pampeanas: sostenibilidad, brechas de conocimiento e integración de políticas. Serie Medio Ambiente y Desarrollo, 118. CEPAL, Santiago de Chile, $65 \mathrm{pp}$.

Pérez Carrera, A.; C.H. Moscuzza y A. Fernández-Cirelli, 2008. Efectos socio-económicos y ambientales de la expansión agropecuaria. Estudio de caso: Santiago del Estero, Argentina. Ecosistemas 17: 5-15.

Pietrarelli, L.T., 2009. Transformación tecnológica-productiva de sistemas agropecuarios de la región central de la Provincia de Córdoba entre 1997 y 2004. Tesis para optar al grado académico de Magíster en Gestión Ambiental Agropecuaria. Universidad Nacional de Córdoba.

Pincén D., E. Viglizzo, L. Carreño y F. Frank, 2010. La relación soja-ecología-ambiente. Entre el mito y la realidad. En: Viglizzo, E. y E. Jobbágy (Editores). Expansión de la frontera agropecuaria en Argentina y su impacto ecológico-ambiental. Ediciones INTA, Buenos Aires. $102 \mathrm{pp}$.

Ramírez, L. y J.C. Porstmann, 2008. Evolución de la frontera agrícola. Campañas 80/81 - 06/07. Revista Agromensajes, Facultad de Ciencias Agrarias de la Universidad Nacional de Rosario. 6 pp.

Salvador V., 2010. Impacto sobre el hábitat. En: Viglizzo, E. y E. Jobbágy (Editores). Expansión de la frontera agropecuaria en Argentina y su impacto ecológicoambiental. Ediciones INTA, Buenos Aires. 102 pp.

Secretaría de Ambiente y Desarrollo Sustentable de la Nación. El avance de la frontera agropecuaria y sus consecuencias. Marzo 2008. www.ambiente.gov.ar/archivos/web/File/032808 avance soja.pdf. Consultado el 10/02/10.

Sociedad Rural de Juárez, 2007. Evaluación de tierras del Partido de Benito Juarez. Estudio técnico contratado al Laboratorio de Geomática de la Unidad Integrada Balcarce INTA-Facultad de Ciencias Agrarias (UNMP).

Torrella, S.; J. Adámoli, P. Herrera y R. Ginzburg, 2005. La expansion agrícola en el Chaco argentino: contrastes entre el litoral fluvial y el interior. En: Temas de biodiversidad del litoral argentino II. F.G. Aceñolaza. INSUGEO. Misceláneas 14:123-134. Tucumán. On Line 1668-3242.

Viglizzo, E., 1994. The response of low-input agricultural systems to environmental variability. A theoretical approach. Agricultural Systems 44: 1-17.

Viglizzo, E., 2008. Capítulo 8. Agricultura, clima y ambiente en Argentina: tendencias, interacciones e impacto. En: Solbrig, O.T. y J. Adámoli (Coordinadores). Agro y 
ambiente: una agenda compartida para el desarrollo sustentable. Foro de la Cadena Agroindustrial Argentina, Buenos Aires.

Viglizzo, E., 2010. El avance de la frontera agropecuaria y el stock de nutrientes (C, N y P). En: Viglizzo, E. y E. Jobbágy (Editores). Expansión de la frontera agropecuaria en Argentina y su impacto ecológico-ambiental. Ediciones INTA, Buenos Aires. 102 pp.

Viglizzo, E. y F. Frank, 2010. Erosión del suelo y contaminación del ambiente. En: Viglizzo, E. y E. Jobbágy (Editores). Expansión de la frontera agropecuaria en Argentina y su impacto ecológicoambiental. Ediciones INTA, Buenos Aires. 102 pp.

Viglizzo, E. y E. Jobbágy (Editores), 2010. Expansión de la frontera agropecuaria en Argentina y su impacto ecológico-ambiental. Ediciones INTA, Buenos Aires. $102 \mathrm{pp}$. 